\title{
Semiclassical Transfer Operator for Complex Built-up Structures
}

\author{
Hanya BEN HAMDIN ${ }^{1 *}$, Gregor TANNER ${ }^{2}$, Stephen CREAGH ${ }^{2}$ \\ 1Sirte University, Science Faculty, Mathematics Department, Sirte, Libya \\ ${ }^{2}$ Nottingham University, Mathematical Sciences School, Nottingham, UK
}

* Corresponding Author : hanyahamdin23@yahoo.com

\section{Keywords}

Bogomolny transfer operator Boundary integral equations Green function

\begin{abstract}
We investigate the wave energy distribution in complex built-up structures with multiple interfaces at which the material properties change discontinuously. We formulate the transfer operator in such a way that it can in principle be made exact, and it is clear where the semiclassical approximations are made at each stage of the derivation. We reformulate the boundary integral equations for the Helmholtz equation in terms of incoming and outgoing boundary waves independently of the boundary conditions and decomposing the green functions into singular and regular components. For demonstration purposes, we apply a semiclassical form of the operator (corresponding to a high-frequency approximation) to polygonal coupled-cavity configurations with abrupt changes of the material properties (such as wave speed and absorption coefficients at the interfaces between the cavities).
\end{abstract}

\section{Introduction}

The transfer operator formalism proposed by Bogomolny [4] has offered a very powerful platform for the application of semiclassical methods to complex quantum and wave problems. Boasman [5] established the connection between the transfer and the boundary integral methods explicitly for quantum billiards, recasting the boundary integral equations as the application of a transfer operator to the wavefunction or its normal derivative on the boundary depending on the prescribed boundary conditions. Subsequent development using Fredholm theory can be found in $[7,8]$, particularly on the Dirichlet case. In this paper we propose that a decomposition of the wavefunction at the boundary into incoming and outgoing components, defined in detail following a separation of the Green function into its regular and singular parts, provides a natural means of establishing this connection beyond leading semiclassical approximations. Semiclassical methods originating in the field of quantum chaos have found widespread application in classical wave problems with complex or chaotic ray limits. A semiclassically-motivated decomposition such as proposed in this paper can then lead to an efficient implementation of fully wave-based calculations. Here we show how to decompose the wave solution at the boundary into a component approaching the boundary and a component leaving it. We propose that the outgoing wave $\psi_{+}(s)$ should be defined simply as the contribution to the boundary integral equation arising from an appropriately defined singular part of the Green function. The simplest definition having the correct semiclassical limit is to define the singular part to correspond to the Green function obtained by replacing the boundary locally by its tangent line or plane. It is referred to this approach as the primitive decomposition and elaborated in [6]. Prosen [9, 10] proposed a more general approach dealing also with smooth potentials, though our approach is more natural because we start from the boundary integral equations (BIEs) and more suitable to accommodate complex built-up structure with multiple interfaces. In this paper we apply the primitive decomposition for the semiclassical coupling of the transfer operator between multiple domains with different local wave number. The transfer operator is derived by combining two operators; the first one is obtained from the relevant BIEs and is called the shift operator. The second operator is obtained from the prescribed boundary conditions and is called the scattering operator. The derivation of the shift operator involves two levels of approximations; the first one is that the derivation of the singular part of the Green operator 
$\widehat{\mathrm{G}}_{0}$ is built on the assumption that the boundary is locally flat. Thus it does not account for the corners of irregular geometries. The second level of approximation is using the asymptotic expansion of the boundary kernels. Having obtained both the shift and the scattering operators semiclassically, we combine them to form the semiclassical transfer operator.

\section{Applications to a Model Problem - Coupled Cavities}

As starting point, we consider a two-cavity configuration in two dimensions shown in Fig. 1. Our aim is a generalisation of the method to large scale, multi-component systems. The considered domain $\Omega=\Omega_{1} \cup \Omega_{2}$ is decomposed into two subdomains with different material properties.

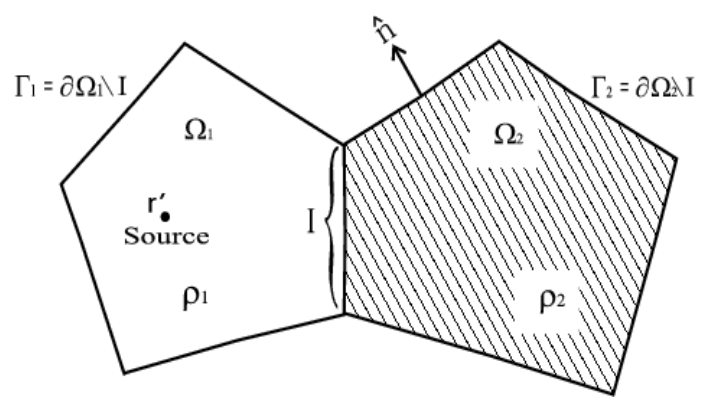

Figure 1. Sketch of the coupled-cavity configuration.

The wavenumber may change discontinuously at the interface $I$ between the left sub-domain $\Omega_{1}$ and the right sub-domain $\Omega_{2}$. We shall denote the external boundary for each sub-domain by,

$\Gamma_{j}=\partial \Omega_{\mathrm{j}} \backslash I, \quad I=\partial \Omega_{1} \cap \partial \Omega_{2}$.

We consider Dirichlet Boundary Conditions (DBCs) along the external boundary $\Gamma_{j}$. To guarantee continuity of the wavefunction and equilibrium of the energy flux across the interface, we impose the following conditions

$$
\psi(s)=\phi(s), \rho_{2} \frac{\partial \psi(s)}{\partial n}=-\rho_{1} \frac{\partial \phi(s)}{\partial n}, \quad s \in I .
$$

The operator $\partial / \partial n$ denotes outward-pointing normal derivative along the boundary of each subdomain. Next we start with the formulation of the transfer operator.

\subsection{The Shift Operator for Coupled-Cavity Configuration}

In higher-dimensional problems, we use a decomposition into singular (near-diagonal) and nonsingular (off-diagonal) parts, as described in [6]. This decomposition is not unique, as there is some freedom in defining the singular part of the Green function. Here we consider the simplest decomposition, first used in [2] and called the primitive decomposition in [6], which separates a singular component using a local Fourier transform along the boundary. In particular we use it to motivate a semiclassical transfer operator equivalent to the formalism used in [4], but which in principle allows systematic development into higher-order and even exact treatments by using more sophisticated developments if the underlying definitions. The Green operators in the BIE for the left sub-domain $\Omega_{1}$ can be written formally as

$$
\begin{aligned}
& \widehat{\mathrm{G}}_{0} \mu(\mathrm{s})=\lim _{\mathrm{x} \rightarrow \mathrm{s}} \int_{\partial \Omega_{1}} \mathrm{G}_{0}\left(\mathrm{x}, s^{\prime} ; \mathrm{k}_{1}\right) \mu\left(s^{\prime}\right) \mathrm{d} s^{\prime}, \\
& \widehat{\mathrm{G}}_{1} \psi(\mathrm{s})=\lim _{\mathrm{x} \rightarrow \mathrm{s}} \int_{\partial \Omega_{1}} \frac{\partial \mathrm{G}_{0}\left(\mathrm{x}, \dot{s}^{\prime} \mathrm{k}_{1}\right)}{\partial n} \psi\left(s^{\prime}\right) \mathrm{d} s^{\prime},
\end{aligned}
$$

in which $x$ denotes a generic point in the interior of $\Omega_{1}$ and $s$ and $s$ label points on its boundary. $\mathrm{G}_{0}\left(\mathrm{x}, x^{\prime} ; \mathrm{k}\right)$ is the free-space Green function satisfying $\left(-\nabla^{2}-\mathrm{k}^{2}\right) \mathrm{G}_{0}\left(\mathrm{x}, \dot{x}^{\prime} ; \mathrm{k}\right)=\delta\left(\mathrm{x}-\mathrm{x}^{\prime}\right)$, while $\Omega_{1}$ is the whole boundary of the left subdomain including the interface. Analogous boundary integral equations are available for $\phi$ and it's normal derivative on the boundary $\partial \Omega_{2}$ of the right sub-domain. We decompose the wavefunctions in the left and right sub-domains in terms of incoming and outgoing waves as,

$\psi=\frac{A}{\sqrt{\hat{P}}}\left(\psi_{-}+\psi_{+}\right), \quad \phi=\frac{B}{\sqrt{\hat{Q}}}\left(\phi_{-}+\phi_{+}\right)$.

where $\mathrm{A}$ and $\mathrm{B}$ are constants, $\psi_{-}$and $\psi_{+}$are respectively the incoming and the outgoing wave components for the left sub-domain, and similarly $\phi_{-}$and $\phi_{+}$for the right sub-domain. The operator $\hat{P}$ is defined formally by

$\hat{P}=\sqrt{1-\hat{p}^{2}}, \quad \hat{p}=\frac{1}{i k_{1}} \frac{\partial}{\partial s}$,

denotes a tangential momentum operator on $\Omega_{1}$. A more concrete definition in terms of a Fourier representation of boundary data on $\Omega_{1}$ is given in [6] but the formal definition above is sufficient for present purposes. In the ray dynamical limit, the operator $\hat{p}$ corresponds to the cosine of the angle of incidence of a ray leaving the boundary, while $\hat{P}$ corresponds to its sine. The operator $\hat{Q}$ is defined analogously on $\Omega_{2}$. The significance of $\hat{P}$ is that a natural decomposition of the Green operator into 
singular and regular parts can be written in terms of it so that

$$
\left(\widehat{G}_{0}\right)^{\operatorname{sing}}=\frac{i}{2 k_{1}} \frac{1}{\hat{P}}, \quad\left(\widehat{G}_{1}\right)^{\text {sing }}=-\frac{1}{2} \hat{I},
$$

where $\hat{I}$ denotes the identity operator. We furthermore let operators $\hat{S}_{0}$ and $\hat{S}_{1}$ be defined so that

$$
\widehat{G}_{0}=\frac{i}{2 k_{1}} \frac{1}{\sqrt{\hat{P}}}\left(\hat{I}+\hat{S}_{0}\right) \frac{1}{\sqrt{\hat{P}}}, \hat{G}_{1}=-\frac{1}{2} \frac{1}{\sqrt{\hat{P}}}\left(\hat{I}+\hat{S}_{1}\right) \sqrt{\widehat{P}}
$$

That is, $\hat{S}_{0}$ and $\hat{S}_{1}$ denote rescaling using $\sqrt{\hat{P}}$ of the regular parts of $\hat{G}_{0}$ and $\hat{G}_{1}$ that are left over once the singular parts defined in (7) are removed. The operators $\hat{S}_{0}$ and $\hat{S}_{1}$ differ slightly from analogously defined operators in [6]: different left and right scaling (by $\sqrt{\widehat{P}}$ ) are used which simplify later calculations in the context of this paper. In the boundary equation for $\psi$, we now let the wave component $\psi_{+}$leaving the boundary be defined by the contribution of the singular parts to the boundary integral equation as follows:

$\frac{A}{\sqrt{\hat{P}}} \psi_{+}=\frac{i}{2 k_{1}} \frac{1}{\hat{P}} \mu+\frac{1}{2} \frac{A}{\sqrt{\hat{P}}}\left(\psi_{-}+\psi_{+}\right)$,

which leads to

$\mu \equiv \frac{\partial \psi}{\partial n}=A i k_{1} \sqrt{\widehat{P}}\left(\psi_{-}-\psi_{+}\right)$.

An analogous calculation in $\Omega_{2}$ leads to

$v \equiv \frac{\partial \phi}{\partial n}=B i k_{2} \sqrt{\hat{Q}}\left(\phi_{-}-\phi_{+}\right)$

The complete boundary integral equation for $\Omega_{1}$ can then be written

$$
\begin{aligned}
& \frac{1}{\sqrt{\hat{P}}}\left(\psi_{-}+\psi_{+}\right)=\frac{i}{2 k_{1}} \frac{1}{\sqrt{\hat{P}}}\left(\hat{I}+\hat{S}_{0}\right) \frac{1}{\sqrt{\hat{P}}}\left[i k _ { 1 } \sqrt { \widehat { P } } \left(\psi_{-}-\right.\right. \\
& \left.\left.\psi_{+}\right)\right]+\frac{1}{2} \frac{1}{\sqrt{\hat{P}}}\left(\hat{I}+\hat{S}_{1}\right) \sqrt{\hat{P}}\left[\frac{1}{\sqrt{\hat{P}}}\left(\psi_{-}+\psi_{+}\right)\right],
\end{aligned}
$$

which can be rearranged to give

$$
\left(2 \hat{I}+\hat{S}_{0}-\hat{S}_{1}\right) \psi_{-}=\left(\hat{S}_{0}+\hat{S}_{1}\right) \psi_{+} .
$$

We now multiply equation (12) from the left by the operator $\left(2 \hat{I}+\hat{S}_{0}-\hat{S}_{1}\right)^{-1}$, to give

$$
\psi_{-}=\left(2 \hat{I}+\hat{S}_{0}-\hat{S}_{1}\right)^{-1}\left(\hat{S}_{0}+\hat{S}_{1}\right) \psi_{+}=\hat{S}^{L} \psi_{+},
$$

where $\hat{S}^{L}$ is the shift operator for the left domain $\Omega_{1}$. A shift operator $\hat{S}^{R}$ for the right sub-domain $\Omega_{2}$ is defined similarly. The inverse operator $\left(2 \hat{I}+\hat{S}_{0}-\hat{S}_{1}\right)^{-1}$ may be difficult to evaluate in exact calculation. To facilitate the computations, we thus employ semiclassical approximations which lead to

$\hat{S}^{L} \approx \hat{S}_{0} \approx \hat{S}_{1}$.

We now collect equation and its analogue for the right sub-domain to form the shift operator

$\left[\begin{array}{l}\psi_{-} \\ \phi_{-}\end{array}\right]=\left[\begin{array}{cc}\hat{S}^{L} & \mathbf{0} \\ \mathbf{0} & \hat{S}^{R}\end{array}\right]\left[\begin{array}{l}\psi_{+} \\ \phi_{+}\end{array}\right]$,

for the coupled-cavity configuration. Using the partition of the boundary of each sub-domain (1), equation (15) can be written as,

$\left(\begin{array}{c}\chi_{\Gamma_{1}} \psi_{-} \\ \chi_{I} \psi_{-} \\ \chi_{I} \phi_{-} \\ \chi_{\Gamma_{2}} \phi_{-}\end{array}\right)=\left(\begin{array}{cccc}\hat{S}_{\Gamma_{1} \Gamma_{1}} & \hat{S}_{\Gamma_{1} I} & \mathbf{0} & \mathbf{0} \\ \hat{S}_{I \Gamma_{1}} & \hat{S}_{I I}^{(1)} & \mathbf{0} & \mathbf{0} \\ \mathbf{0} & \mathbf{0} & \hat{S}_{I I}^{(2)} & \hat{S}_{I \Gamma_{2}} \\ \mathbf{0} & \mathbf{0} & \hat{S}_{\Gamma_{2} I} & \hat{S}_{\Gamma_{2} \Gamma_{2}}\end{array}\right)\left(\begin{array}{c}\chi_{\Gamma_{1}} \psi_{+} \\ \chi_{I} \psi_{+} \\ \chi_{I} \phi_{+} \\ \chi_{\Gamma_{2}} \phi_{+}\end{array}\right)$

where $\chi$ is the characteristic function and defined by

$\chi_{D}(s)= \begin{cases}1, & \text { if } s \in D \\ 0, & \text { otherwise }\end{cases}$

We distinguish here between $\hat{S}_{I I}^{(1)}$ and $\hat{S}_{I I}^{(2)}$, the interface-to-interface blocks of the shift operator computed respectively from the left and right sides. Although they are constructed on the same boundary segment, the normal derivatives point in opposite directions in each case, so the corresponding blocks are not identical. Note, however, that for the examples used to illustrate the procedure in this paper, where the interface is planar, we will find that $\hat{S}_{I I}^{(1)}$ and $\hat{S}_{I I}^{(2)}$ II both vanish at leading order. Equation (16) can be cast as,

$\Psi_{-}=\hat{S} \Psi_{+}$,

where $\hat{S}$ is the shift operator for the coupled-cavity configuration. Hereafter, we use the following notation for the outgoing wave vector,

$\Psi_{+}=\left(\begin{array}{c}\chi_{\Gamma_{1}} \psi_{+} \\ \chi_{I} \psi_{+} \\ \chi_{I} \phi_{+} \\ \chi_{\Gamma_{2}} \phi_{+}\end{array}\right)=\left(\begin{array}{c}\psi_{+}^{L} \\ \psi_{+}^{I} \\ \phi_{+}^{I} \\ \phi_{+}^{R}\end{array}\right)$

and similarly for the incoming wave vector $\Psi_{-}$. The functions $\psi_{+}^{L}$ and $\psi_{+}^{I}$ are respectively the incoming wave sub-vector along the outer boundary of the left sub-domain and the interface, and similarly for 
$\phi_{+}^{R}$ and $\phi_{+}^{I}$ for the right sub-domain. Next we derive the operator which incorporates the boundary conditions.

\subsection{The Scattering Operator}

We next present a derivation of a scattering operator which encodes the boundary conditions for the coupled-cavity configuration. This operator tells us how an incoming wave is scattered once it hits any part of the boundary of the coupled cavities. We set DBCs along the external boundary (excluding the interface) of the coupled cavities. That is,

$\chi_{\Gamma_{1}} \psi(s)=0, \quad \chi_{\Gamma_{2}} \phi(s)=0$.

The function $\chi_{\Gamma_{1}}$ denotes the characteristic function defined by Eq. (17). Further to the DBCs along the external boundary $\Gamma_{j}$, one needs to impose the continuity conditions of the wavefunction and the equilibrium of the energy flux normal to the interface as given by the following equations,

$\chi_{I} \psi(s)=\chi_{I} \phi(s), \rho_{2} \chi_{I} \frac{\partial \psi(s)}{\partial n_{s}}=-\rho_{1} \chi_{I} \frac{\partial \phi(s)}{\partial n_{s}}$.

The constants $\rho_{1}$ and $\rho_{2}$ are the material densities of the left and right sub-domains, respectively. Turning these boundary conditions into relationships between the incoming and outgoing wave components is nontrivial in exact calculation because the characteristic functions in equations (20) and (21) do not commute with the $\widehat{P}$ and $\hat{Q}$ operators that appear in equations (5), (9) and (10). We overcome this by passing immediately to a semiclassical approximation in which diffraction effects arising from wave incidence on corners are ignored. Inserting the DBCs (20) into the decomposition relations given by equation (5) leads to the following relations,

$\chi_{\Gamma_{1}} \psi_{+}=-\chi_{\Gamma_{1}} \psi_{-}, \quad \chi_{\Gamma_{2}} \phi_{+}=-\chi_{\Gamma_{2}} \phi_{-}$,

where at leading order in semiclassical approximation we have neglected the commutator between $\chi_{I}$ and the operators $1 / \sqrt{\hat{P}}$ and $1 / \sqrt{\hat{Q}}$. That is, we have neglected contributions from

$C=\left[\chi_{I}, \frac{1}{\sqrt{\hat{P}}}\right]$

that in the exact calculation would contribute distributional terms corresponding to diffraction from the associated corners. It is shown in [2] that contributions from the commutator (23) decay in the semiclassical limit $\left(k_{1} \rightarrow \infty\right)$ and may be neglected at leading order. We should also incorporate the continuity conditions along the interface. We insert the decomposition relations given by equation (5) into the first line of the conditions described by equation (21). One then has

$$
A \chi_{I} \frac{1}{\sqrt{\hat{P}}}\left(\psi_{-}+\psi_{+}\right)=B \chi_{I} \frac{1}{\sqrt{\hat{Q}}}\left(\phi_{-}+\phi_{+}\right) .
$$

The constants $A=\sqrt{\rho_{1} / k_{1}}$ and $B=\sqrt{\rho_{2} / k_{2}}$ are chosen to emphasise conservation of energy flux across the interface in the dissipationless limit: scattering into reflected and transmitted amplitudes in a ray limit is then described by a unitary matrix in semiclassical approximation. Similarly, the boundary conditions for the normal derivatives give $B \chi_{I} \sqrt{\widehat{P}}\left(\psi_{-}-\psi_{+}\right)=A \chi_{I} \sqrt{\widehat{Q}}\left(\phi_{+}-\phi_{-}\right)$.

As with the boundary conditions placed on $\Gamma_{1}$ and $\Gamma_{2}$, an exact treatment requires us to account for the non-commutation of the operators $\widehat{P}$ and $\hat{Q}$ with $\chi_{I}$. At leading order in semiclassical approximation, however, and in particular neglecting diffractive effects associated with scattering from the endpoints of $I$, we can represent these equations after some manipulation in the form

$\chi_{I} \psi_{+} \approx \hat{r} \chi_{I} \psi_{-}+\hat{t} \chi_{I} \phi_{-}, \chi_{I} \phi_{+} \approx-\hat{r} \chi_{I} \phi_{-}+\hat{t} \chi_{I} \psi_{-}$,

where

$\hat{r}=\chi_{I}\left(\frac{B^{2} \hat{P}-A^{2} \hat{Q}}{B^{2} \hat{P}+A^{2} \hat{Q}}\right) \chi_{I}$,

and

$\hat{t}=\chi_{I}\left(\frac{2 A B \sqrt{\hat{P}} \hat{Q}}{B^{2} \hat{P}+A^{2} \hat{Q}}\right) \chi_{I}$,

represent projections onto the interval $I$ of reflection and transmission operators. We now combine equations (22), and (25) to form the scattering operator as the following,

$$
\left(\begin{array}{c}
\psi_{+}^{L} \\
\psi_{+}^{I} \\
\phi_{+}^{I} \\
\phi_{+}^{R}
\end{array}\right)=\left(\begin{array}{cccc}
-\hat{I} & \mathbf{0} & \mathbf{0} & \mathbf{0} \\
\mathbf{0} & \hat{r} & \hat{t} & \mathbf{0} \\
\mathbf{0} & \hat{t} & -\hat{r} & \mathbf{0} \\
\mathbf{0} & \mathbf{0} & \mathbf{0} & -\hat{I}
\end{array}\right)\left(\begin{array}{c}
\psi_{-}^{L} \\
\psi_{-}^{I} \\
\phi_{-}^{I} \\
\phi_{-}^{R}
\end{array}\right)
$$

This equation can be cast in the form

$\Psi_{+}=\hat{R} \Psi_{-}$.

The map $\hat{R}$ will be referred to as the scattering operator representing reflection and transmission of wave incident on the boundary into waves moving away from it. Diagonal blocks correspond to 
reflection while the offdiagonal blocks correspond to transmission across the interface $I$ from one domain into the other.

\subsection{The Semiclassical Transfer Operator}

To summarise, in the previous sections we obtained both the shift and the scattering operator semiclassically. We now combine them to construct the semiclassical transfer operator $\widehat{T}$. Inserting the outgoing vector given by equation (29) into equation (18) and using equation (19), leads to

$\left(\begin{array}{l}\psi_{-}^{L} \\ \psi_{-}^{I} \\ \phi_{-}^{I} \\ \phi_{-}^{R}\end{array}\right)=$
$\left(\begin{array}{cccc}\hat{S}_{\Gamma_{1} \Gamma_{1}} & \hat{S}_{\Gamma_{1} I} & \mathbf{0} & \mathbf{0} \\ \hat{S}_{I \Gamma_{1}} & \hat{S}_{I I}^{(1)} & \mathbf{0} & \mathbf{0} \\ \mathbf{0} & \mathbf{0} & \hat{S}_{I I}^{(2)} & \hat{S}_{I \Gamma_{2}} \\ \mathbf{0} & \mathbf{0} & \hat{S}_{\Gamma_{2} I} & \hat{S}_{\Gamma_{2} \Gamma_{2}}\end{array}\right)\left(\begin{array}{cccc}-\hat{I} & \mathbf{0} & \mathbf{0} & \mathbf{0} \\ \mathbf{0} & \hat{r} & \hat{t} & \mathbf{0} \\ \mathbf{0} & \hat{t} & -\hat{r} & \mathbf{0} \\ \mathbf{0} & \mathbf{0} & \mathbf{0} & -\hat{I}\end{array}\right)\left(\begin{array}{l}\psi_{-}^{L} \\ \psi_{-}^{I} \\ \phi_{-}^{I} \\ \phi_{-}^{R}\end{array}\right)$.

This equation can be rearranged as the following,

$$
\left(\begin{array}{l}
\psi_{-}^{L} \\
\psi_{-}^{I} \\
\phi_{-}^{I} \\
\phi_{-}^{R}
\end{array}\right)=\left(\begin{array}{cccc}
\hat{T}_{\Gamma_{1} \Gamma_{1}}^{d} & \hat{T}_{\Gamma_{1} I}^{r} & \hat{T}_{\Gamma_{1} I}^{t} & \mathbf{0} \\
\widehat{T}_{I \Gamma_{1}}^{d} & \widehat{T}_{I I}^{(1) r} & \widehat{T}_{I I}^{(1) t} & \mathbf{0} \\
\mathbf{0} & \widehat{T}_{I I}^{(2) t} & \widehat{T}_{I I}^{(2) r} & \widehat{T}_{I \Gamma_{2}}^{d} \\
\mathbf{0} & \widehat{T}_{\Gamma_{2} I}^{t} & \widehat{T}_{\Gamma_{2} I}^{r} & \widehat{T}_{\Gamma_{2} \Gamma_{2}}^{d}
\end{array}\right)\left(\begin{array}{c}
\psi_{-}^{L} \\
\psi_{-}^{I} \\
\phi_{-}^{I} \\
\phi_{-}^{R}
\end{array}\right),
$$

where the superscripts $d, r$ and $t$ refer to contributions to the transfer operator from crossings of the interior which follow reflection from $\Gamma_{i}$, reflection from $I$ and transmission across $I$, respectively. This equation can be cast in the form

$\Psi_{-}=\widehat{T} \Psi_{-}$.

The map $\hat{T}$ will be referred to as semiclassical transfer operator.

\section{Conclusion}

In this paper we semiclassically formulated the transfer operator for coupled cavities with different local wavenumbers changing at the interfaces between them. We point out the semiclassical approximations which have been made at each stage of the derivation. The semiclassical transfer operator was derived where the physical solution is an eigenfunction, however an analogous formulation can be done for the Green function [2]. These results can be improved by considering the treatment of corners when defining the singular part of the Green function, that is, including the corners diffraction effects. Also it can be improved by considering the commutator contribution (23) in the derivation of the scattering operator and performing the operator inversion in equation (13) in the derivation of the shift operator.

\section{Acknowledgement}

HBH acknowledges support by the Libyan Ministry of Education. SCC acknowledges support from EPSRC under grant no. EP/F036574/1. Further support by the EU (FP7 IAPP grant MIDEA) is gratefully acknowledged.

\section{References}

[1] M. Abramowitz, I. A. Stegun "Handbook of Mathematical Functions" Dover, New York (1972)

[2] H. Ben Hamdin "Boundary element and transfer operator methods for multi-component wave systems" PhD Thesis, School of Mathematical Sciences, Nottingham University, UK( 2012)

[3] H. Ben Hamdin, G. Tanner "Multi-component BEM for the Helmholtz equation - A normal derivative method" IOS Press, Shock and Vibration, 19 (2012) 957-967

[4] E.B. Bogomolny "Semiclassical quantization of multidimensional systems" Nonlinearity, 5(1992) 805-866

[5] P. A. Boasman "Semiclassical Accuracy for Billiards" Nonlinearity, 7 (1994) 485

[6] S. C. Creagh, H. Ben Hamdin and G. Tanner "In-out decomposition of boundary integral equations" J.Phys. A: Math. Theor., 46(2013)

[7] B. Georgeot, R. E. Prange "Exact and Quasiclassical Fredholm Solutions of Quantum Billiards" Phys. Rev. Lett.,74 (15) (1992) 2851-2854

[8] B. Georgeot, R. E. Prange "Fredholm Theory for Quasiclassical Scattering" Phys. Rev. Lett.,74 (21) (1995) 4110-4113

[9] T. Prosen "Exact quantum surface of section method" J. Phys. A:Math. Gen., 27(1994)L709L714

[10] T. Prosen "General quantum surface-of-section method” J. Phys. A:Math. Gen., 28(1995) 4133-4155 